\title{
REPRESENTATIONS OF QUANTUM GROUPS AND (CONDITIONALLY) INVARIANT $q$-DIFFERENCE EQUATIONS
}

\author{
VLADIMIR K. DOBREV \\ Bulgarian Academy of Sciences \\ Institute of Nuclear Research and Nuclear Energy \\ 72 Tsarigradsko Chaussee, 1784 Sofia, Bulgaria \\ E-mail: dobrev@bgearn.acad.bg,dobrev@inrne.acad.bg
}

\begin{abstract}
We give a systematic discussion of the relation between $q$-difference equations which are conditionally $U_{q}(\mathcal{G})$-invariant and subsingular vectors of Verma modules over $U_{q}(\mathcal{G})$ (the Drinfeld-Jimbo $q$-deformation of a semisimple Lie algebra $\mathcal{G}$ over $\mathbb{C}$ or $\mathbb{R}$ ). We treat in detail the cases of the conformal algebra, $\mathcal{G}=s u(2,2)$, and its complexification, $\mathcal{G}=s l(4)$. The conditionally invariant equations are the $q$-deformed d'Alembert equation and a new equation arising from a subsingular vector proposed by Bernstein-Gel'fand-Gel'fand.
\end{abstract}

1. Introduction. It is well known that the d'Alembert equation

$$
\square f(x)=0
$$

is Poincaré and even conformal invariant, cf., e.g., [1]. Here $f(x)$ is a scalar field of fixed conformal weight, $x=\left(x_{0}, x_{1}, x_{2}, x_{3}\right)$ denotes the Minkowski space-time coordinates, and $\square$ is the d'Alembert operator: $\square=\partial^{\mu} \partial_{\mu}=(\vec{\partial})^{2}-\left(\partial_{0}\right)^{2}$.

In the present paper we would like to present representation-theoretic reinterpretations of this fact. There are two aspects of this. First, from the point of view of induced representations of the conformal algebra $s u(2,2)$ one can not automatically obtain representations which are also irreducible finite-dimensional (e.g., scalar above) representations of the Lorentz subalgebra. To ensure this one has to impose additional conditions and to restrict to functions which obey these conditions. In the case at hand there are two such conditions and it is on such functions that (1.1) is conformal invariant. That is why we shall call the conformal invariance of (1.1) conditional. (Using approaches different from ours other conditionally invariant equations were considered in [20], [21], [3], [17], [16], (for some more comment see subsection 3.1).)

1991 Mathematics Subject Classification: Primary 17B35; Secondary 81R05, 22E47.

The paper is in final form and no version of it will be published elsewhere. 
The second aspect is that we can find a counterpart of (1.1) in the representation theory of Verma modules over the complexification $s l(4)$ of $s u(2,2)$. Namely, this counterpart is a subsingular vector of a Verma module (definition below).

In the present paper we consider (1.1) and conditional invariant equations in general applying the approach of [6]. The needed results from [6] stated in condensed form (and in some detail in subsection 3.1) are: to every singular, resp., subsingular vector of a Verma module over a semi-simple (also reductive) Lie algebra $\mathcal{G}$ there corresponds a differential operator and equation invariant, resp., conditionally invariant with respect to $\mathcal{G}$. (Both statements are valid also for the corresponding Lie group with some additional subtleties, cf. [6].)

One of the specifics of the approach of [6] is that if one wants to consider (conditional) invariance with respect to some real Lie algebra $\mathcal{G}_{0}$ one has also to know the invariance with respect to the complexification $\mathcal{G}$ of $\mathcal{G}_{0}$. The same is true in the $q$-deformed case. That is why we treat in parallel $s l(4)$ and the conformal algebra $s u(2,2)$, and analogously $U_{q}(\operatorname{sl}(4))$ and $U_{q}(s u(2,2))$.

We treat in detail the $q=1$ case since some of our results are new also in this classical situation. In particular, we give also (1.1) with nontrivial right hand side and we present a new conditionally invariant equation.

The paper is organized as follows. The notion of subsingular vector is explained in Subsection 2.1 for arbitrary ( $q$-deformed) simple Lie algebras. Then we restrict to $s l(4)$ and $U_{q}(s l(4))$ and we give the singular and subsingular vectors we shall need. In parallel we give the explicit conditions for irreducibility of the lowest weight modules. Here the exposition is common for generic $q$. These results are applied in Sections 3, resp. 4, to get explicitly the conditionally invariant equations for $q=1$, resp. for generic $q$.

\section{Subsingular vectors}

2.1. Let $\mathcal{G}=\mathcal{G}^{+} \oplus \mathcal{H} \oplus \mathcal{G}^{-}$be a semisimple Lie algebra, where $\mathcal{H}$ is a Cartan subalgebra of $\mathcal{G}, \mathcal{G}^{+}$, resp., $\mathcal{G}^{-}$, are the positive, resp., negative root vector spaces of the root system $\Delta=\Delta(\mathcal{G}, \mathcal{H})$, corresponding to the decomposition $\Delta=\Delta^{+} \cup \Delta^{-}$into positive and negative roots. Let $\Delta_{S}=\left\{\alpha_{i} \mid i=1, \ldots, r=\operatorname{rank} \mathcal{G}\right\}$ be the system of simple roots of $\Delta$. We use the standard deformation $U_{q}(\mathcal{G})$ [15], [18] given in terms of the Chevalley generators $X_{i}^{ \pm}, H_{i} \in \mathcal{H}, i=1, \ldots, r$ of $\mathcal{G}$. The elements $H_{i}$ span the Cartan subalgebra $\mathcal{H}$ of $\mathcal{G}$, while the elements $X_{i}^{ \pm}$generate the subalgebras $U_{q}\left(\mathcal{G}^{ \pm}\right)$.

A lowest weight module $(\mathrm{LWM}) M^{\Lambda}$ over $U_{q}(\mathcal{G})$ is given by the lowest weight $\Lambda \in \mathcal{H}^{*}$ $\left(\mathcal{H}^{*}\right.$ is the dual of $\left.\mathcal{H}\right)$ and a lowest weight vector $v_{0}$ so that $X v_{0}=0$ if $X \in \mathcal{G}^{-}$, $H v_{0}=\Lambda(H) v_{0}$ if $H \in \mathcal{H}$. In particular, we use the Verma modules $V^{\Lambda}$ over $U_{q}(\operatorname{sl}(\mathcal{G}))$ which are the lowest weight modules such that $V^{\Lambda} \cong U_{q}\left(\mathcal{G}^{+}\right) v_{0}$.

Let us introduce the numbers:

$$
m_{i}=m_{i}(\Lambda) \doteq(\rho-\Lambda)\left(H_{i}\right)=1-\Lambda\left(H_{i}\right)=1-\left(\Lambda, \alpha_{i}^{\vee}\right), \quad i=1, \ldots, r
$$

where $\rho=\frac{1}{2} \sum_{\beta \in \Delta^{+}} \beta,\left(\rho\left(H_{k}\right)=\left(\rho, \alpha_{k}\right)=1\right),(\cdot, \cdot)$ is the scalar product of the roots normalized so that for the short roots $\alpha$ we have $(\alpha, \alpha)=2, \alpha^{\vee} \equiv 2 \alpha /(\alpha, \alpha)$.

We note that these numbers completely determine the lowest weight $\Lambda$ and shall be 
used also for the characterization of the LWM. The collection of these numbers shall be called the signature of $\Lambda$ and denoted $\chi(\Lambda)$ or just $\chi$ :

$$
\chi=\chi(\Lambda) \doteq\left(m_{1}, \ldots, m_{r}\right)
$$

Analogously, we shall also use numbers corresponding to arbitrary positive roots :

$$
m_{\alpha}=m_{\alpha}(\Lambda) \doteq(\rho-\Lambda)\left(H_{\alpha}\right)=\left(\rho-\Lambda, \alpha^{\vee}\right), \quad \alpha \in \Delta^{+}
$$

where $H_{\alpha} \in \mathcal{H}$ corresponds to the root $\alpha$ by the isomorphism $\mathcal{H} \cong \mathcal{H}^{*}$, (as $H_{i}$ corresponds to $\alpha_{i}$ ). Certainly, each $m_{\alpha}$ is a fixed linear combination of $m_{i}$, however, these numbers have independent importance as we shall see just below. Naturally, $m_{\alpha_{i}}=m_{i}$.

In the present paper we restrict to the case when the deformation parameter $q$ is not a nontrivial root of 1 . (For the case when $q$ is a nontrivial root of 1 we refer to [7].) In this case a Verma module $V^{\Lambda}$ is reducible [2] $(q=1)$, [7] iff at least one of the numbers $m_{\alpha}$ is a positive integer:

$$
m_{\alpha} \in \mathbb{N}
$$

Whenever (2.4) is fulfilled there exists a singular vector $v_{s}=v^{\alpha, m_{\alpha}}$ in $V^{\Lambda}$ such that $v_{s} \notin \mathbb{C} v_{0}, X v_{s}=0, \forall X \in \mathcal{G}^{-}$and $H v_{s}=\left(\Lambda+m_{\alpha} \alpha\right)(H) v_{s}, \forall H \in \mathcal{H}$. The space $I^{\alpha}=$ $U_{q}\left(\mathcal{G}^{+}\right) v^{\alpha, m_{\alpha}}$ is a proper submodule of $V^{\Lambda}$ isomorphic to the Verma module $V^{\Lambda+m_{\alpha} \alpha}$ with a shifted lowest weight $\Lambda+m_{\alpha} \alpha$ [6], [7]. Clearly, this implies that $V^{\Lambda}$ and $V^{\Lambda+m_{\alpha} \alpha}$ have the same values of the Casimir operators.

Remark 1 . Note that if we were considering highest instead of lowest weights, the analog of the numbers $m_{k},\left(m_{\alpha}\right)$, would be defined as $m_{k}^{\text {h.w. }}=1+\Lambda\left(H_{i}\right),\left(m_{\alpha}^{\text {h.w. }}=(\rho+\right.$ $\left.\Lambda)\left(H_{\alpha}\right)\right)$; the shifted weight is $\Lambda-m_{\alpha}^{\text {h.w. }} \alpha$. However, the statement about the reducibility is unchanged. [6]. $\diamond$

It is important that one can find explicit formulae for the singular vectors. The singular vector introduced above is given by [6], [7]:

$$
v_{s}=v^{\alpha, m_{\alpha}}=\mathcal{P}^{\alpha, m_{\alpha}}\left(X_{1}^{+}, \ldots, X_{r}^{+}\right) v_{0}
$$

where $\mathcal{P}^{\alpha, m_{\alpha}}$ is a homogeneous polynomial in its variables of degrees $m n_{i}$, where $n_{i} \in \mathbb{Z}_{+}$ come from $\alpha=\sum n_{i} \alpha_{i}, \alpha_{i}$ - the system of simple roots. The polynomial $\mathcal{P}^{\alpha, m_{\alpha}}$ is unique up to a non-zero multiplicative constant. The papers [6], [7] contain all explicit singular vectors needed in the present paper. Note that we refer to both, since [6] gives formulae for $q=1$, while [7] gives such formulae for general $q$. (More general explicit formulae for singular vectors, including all singular vectors for $U_{q}(s l(n))$, are contained in [8]. Note that the modules considered in [7], [8] are highest weight modules and the singular vectors are polynomials in $X_{i}^{-}$; the translation of those formulae to the lowest weight module setting is straightforward in view of the above Remark.)

Certainly, (2.4) may be fulfilled for several positive roots (even for all of them). Let $\Delta_{\Lambda}$ denote the set of all positive roots for which (2.4) is fulfilled, and let us denote: $\tilde{I}^{\Lambda} \equiv \cup_{\alpha \in \Delta_{\Lambda}} I^{\alpha}$. Clearly, $\tilde{I}^{\Lambda}$ is a proper submodule of $V^{\Lambda}$. Let us also denote $F^{\Lambda} \equiv V^{\Lambda} / \tilde{I}^{\Lambda}$.

Further we shall use also the following notion. The singular vector $v_{1}$ is called descendant of the singular vector $v_{2} \notin \mathbb{C} v_{1}$ if there exists a homogeneous polynomial $P_{12}$ in $X_{i}^{+}$ 
so that $v_{1}=P_{12} v_{2}$. Clearly, in this case we have: $I^{1} \subset I^{2}$, where $I^{k}$ is the submodule generated by $v_{k}$.

The Verma module $V^{\Lambda}$ contains a unique proper maximal submodule $I^{\Lambda}\left(\supseteq \tilde{I}^{\Lambda}\right)[2]$, [4]. Among the lowest weight modules with lowest weight $\Lambda$ there is a unique irreducible one, denoted by $L_{\Lambda}$, i.e., $L_{\Lambda}=V^{\Lambda} / I^{\Lambda}$. (If $V^{\Lambda}$ is irreducible then $L_{\Lambda}=V^{\Lambda}$.)

It may happen that the maximal submodule $I^{\Lambda}$ coincides with the submodule $\tilde{I}^{\Lambda}$ generated by all singular vectors. This is, e.g., the case for all Verma modules if rank $\mathcal{G} \leq 2$, or when (2.4) is fulfilled for all simple roots (and, as a consequence for all positive roots). Here we are interested in the cases when $\tilde{I}^{\Lambda}$ is a proper submodule of $I^{\Lambda}$. We need the following notion.

Definition. Let $V^{\Lambda}$ be a reducible Verma module. A vector $v_{\mathrm{su}} \in V^{\Lambda}$ is called a subsingular vector if $v_{\mathrm{su}} \notin \tilde{I}^{\Lambda}$ and the following holds:

$$
X v_{\mathrm{su}} \in \tilde{I}^{\Lambda}, \quad \forall X \in \mathcal{G}^{-}
$$

Remark 2. The image of a subsingular vector in the factor-module $F^{\Lambda}$ is a singular vector of $F^{\Lambda}$. For shortness we shall say the subsingular vector 'becomes' a singular vector in the corresponding factor-module. From this it is also clear that a subsingular vector may be represented by a homogeneous polynomial in $X_{i}^{+} \cdot \diamond$

We need to be more explicit even in the general case. First of all it is clear that it is enough for a vector to be subsingular if (2.6) holds for the negative simple root vectors $X_{i}^{-}$. We can rewrite $(2.6)$ in the following way:

$$
X_{i}^{-} v_{\mathrm{su}}=\sum_{\alpha \in \Delta_{i}} Q_{i \alpha} v^{\alpha, m_{\alpha}}
$$

where $Q_{i \alpha}$ are homogeneous polynomials such that the RHS is a homogeneous polynomial, and $\Delta_{i}$ is a subset of $\Delta_{\Lambda} \subset \Delta^{+}$, such that $\alpha \in \Delta_{i}$ iff $Q_{i \alpha}$ is a nonzero polynomial. Let us denote by $\Delta_{\mathrm{su}}$ the union of $\Delta_{i}: \Delta_{\mathrm{su}} \equiv \cup_{i=1}^{r} \Delta^{i}$. We shall call $\Delta_{\mathrm{su}}$ the set of roots associated with the subsingular vector $v_{\mathrm{su}}$. The corresponding set of singular vectors $\left\{v^{\alpha, m_{\alpha}} \mid \alpha \in \Delta_{\text {su }}\right\}$ will be called singular vectors associated with the subsingular vector $v_{\text {su }}$. Clearly $\Delta_{\mathrm{su}}$ is a subset of $\Delta_{\Lambda}$ and in general a proper subset. Let $I_{\mathrm{su}} \equiv \cup_{\alpha \in \Delta_{\mathrm{su}}} I^{\alpha}\left(\subseteq \tilde{I}^{\Lambda}\right)$, $F_{\text {su }} \equiv V^{\Lambda} / I_{\text {su }}$; then $v_{\text {su }}$ becomes a singular vector in $F_{\text {su }}$, i.e., when we factorize all singular vectors associated with it.

Clearly, if two singular vectors $v_{1}$ and $v_{2}$ belong to $\Delta_{\Lambda}\left(\Delta_{i}, \Delta_{\text {su }}\right)$ and $v_{1}$ is descendant of $v_{2}$, then we can omit $v_{1}$ from the set $\Delta_{\Lambda}\left(\Delta_{i}, \Delta_{\mathrm{su}}\right)$.

Clearly, $v_{\text {su }}$ and $\tilde{I}^{\Lambda}$ generate a submodule $I_{\text {su }}^{\Lambda}$ so that:

$$
I_{\mathrm{su}} \subseteq \tilde{I}^{\Lambda} \subset I_{\mathrm{su}}^{\Lambda} \subseteq I^{\Lambda} \subset V^{\Lambda}
$$

2.2. We restrict now to $\mathcal{G}=s l(4)$, (cf. [9], [10]). For the six positive roots of the root system of $\operatorname{sl}(4)$ one has from $(2.1),(2.3)$ (cf. [6]):

$$
\begin{aligned}
m_{1} & =1-\Lambda\left(H_{1}\right) \\
m_{2} & =1-\Lambda\left(H_{2}\right) \\
m_{3} & =1-\Lambda\left(H_{3}\right) \\
m_{12} & =2-\Lambda\left(H_{12}\right)=m_{1}+m_{2}
\end{aligned}
$$




$$
\begin{aligned}
& m_{23}=2-\Lambda\left(H_{23}\right)=m_{2}+m_{3} \\
& m_{13}=3-\Lambda\left(H_{13}\right)=m_{1}+m_{2}+m_{3}
\end{aligned}
$$

Thus the signature here is : $\chi=\left(m_{1}, m_{2}, m_{3}\right)$.

For further reference we give the value of the $s l(4)$ second order Casimir operator [22] in terms of the above notation:

$$
C_{2}=\frac{1}{2}\left(m_{13}^{2}+m_{2}^{2}+\frac{1}{2}\left(m_{1}-m_{3}\right)^{2}\right)-5
$$

which is normalized to take zero value on the trivial irrep $\left(m_{k}=1\right)$ (and thus on all representations partially equivalent to it).

In the case of $s l(4)$ and $U_{q}(s l(4))$ there are two generic situations to have subsingular vectors. This follows from general results of [19] and is analyzed in detail (from the mathematical point of view) in [13]. We discuss examples of subsingular vectors from both situations in the next subsections.

2.3. Here we treat the Bernstein-Gel'fand-Gel'fand example of a subsingular vector which appeared in the seminal paper [2] $(q=1)$ and which we give for general $q$. It occurs for $\Lambda\left(H_{1}\right)=\Lambda\left(H_{3}\right)=1, \Lambda\left(H_{2}\right)=0$, i.e., $\chi=\left(m_{1}, m_{2}, m_{3}\right)=(0,1,0)$. Thus there are four positive $m_{\alpha} \in \mathbb{I N}$ from (2.9) : $m_{2}=m_{12}=m_{23}=m_{13}=1$. Correspondingly, there are four singular vectors:

$$
\begin{aligned}
& v_{2}=X_{2}^{+} v_{0}, \quad m_{2}=1 \\
& v_{12}^{\prime}=X_{1}^{+} X_{2}^{+} v_{0}=X_{1}^{+} v_{2}, \quad m_{12}=1 \\
& v_{23}^{\prime}=X_{3}^{+} X_{2}^{+} v_{0}=X_{3}^{+} v_{2}, \quad m_{23}=1 \\
& v_{13}^{\prime}=X_{1}^{+} X_{3}^{+} X_{2}^{+} v_{0}=X_{1}^{+} X_{3}^{+} v_{2}, \quad m_{13}=1
\end{aligned}
$$

However, only the singular vector $v_{2}$ is relevant since the others are its descendants.

What is important for us is that there is the following subsingular vector:

$$
v_{\mathrm{bgg}}=\left(X_{1}^{+} X_{2}^{+} X_{3}^{+}-X_{3}^{+} X_{2}^{+} X_{1}^{+}\right) v_{0}
$$

It is easy to see:

$$
\begin{aligned}
& X_{1}^{-} v_{\mathrm{bgg}}=v_{23}^{\prime}=X_{3}^{+} v_{2} \\
& X_{2}^{-} v_{\mathrm{bgg}}=0 \\
& X_{3}^{-} v_{\mathrm{bgg}}=-v_{12}^{\prime}=-X_{1}^{+} v_{2}
\end{aligned}
$$

thus, indeed, (2.6) is fulfilled, while comparing with (2.7) we see that indeed $v_{2}$ is associated with $v_{\text {bgg }}$.

It is useful for future applications to have different expression for the subsingular vectors. Formula (2.12a) is in the unordered Chevalley basis. An expression in the ordered PBW basis is:

$$
v_{\mathrm{bgg}}=\left(X_{13}^{+}+q X_{3}^{+} X_{12}^{+}+q^{-1} X_{23}^{+} X_{1}^{+}\right) v_{0}
$$

which for $q=1$ is exactly equal to $(2.12 a)$ and for $q \neq 1$ differs from $(2.12 a)$ by the inessential term $\left(q-q^{-1}\right) X_{1}^{+} X_{3}^{+} X_{2}^{+} v_{0} \in \tilde{I}^{\Lambda}$. For $q=1$ a third expression coinciding 
with $(2.12 a, b)$ is:

$$
v_{\mathrm{bgg}}=\left(X_{12}^{+} X_{3}^{+}+X_{23}^{+} X_{1}^{+}\right) v_{0}, \quad q=1
$$

Note that we have translated the result of [2] into our lowest weight module setting and that the actual expression for $v_{\text {bgg }}$ in [2], (given naturally for $q=1$ ), is not correct. (Also formulae $(2.11 b)$ are not given in [2].)

Let $\widetilde{|2\rangle}$ denote the lowest weight vector of the factor-module $F_{2}=V^{\Lambda} / I_{2}$. Then the singular vectors in (2.11) become null-conditions, the relevant one $(2.11 a)$ giving:

$$
X_{2}^{+} \widetilde{|2\rangle}=0
$$

Clearly, $v_{\text {bgg }}$ becomes a singular vector in $F_{2}$. If we factor out also $v_{\text {bgg }}$ we have the following null conditions in the resulting irreducible module $L_{\Lambda}$ with lowest weight vector $|2\rangle$ :

$$
\begin{aligned}
X_{2}^{+}|2\rangle & =0 \\
\left(X_{1}^{+} X_{2}^{+} X_{3}^{+}-X_{3}^{+} X_{2}^{+} X_{1}^{+}\right)|2\rangle & =0
\end{aligned}
$$

2.4. In this and the remaining subsections of this Section we consider the other archetypal $s l(4)$ example [19], [13]. In this subsection we give some preliminaries. We take first an arbitrary Verma module $V^{\Lambda}$ and the following vector:

$$
v_{f}=\mathcal{P} v_{0}
$$

where $\mathcal{P}$ is the following homogeneous polynomial in $U_{q}\left(\mathcal{G}^{+}\right)$:

$$
\mathcal{P}=X_{13}^{+} X_{2}^{+}-q^{-1} X_{12}^{+} X_{23}^{+}
$$

Below we shall need the following technical result:

$$
\begin{aligned}
X_{2}^{-} v_{f}= & q^{-1}\left(\left[\Lambda\left(H_{2}\right)+1\right]_{q} X_{2}^{+} X_{1}^{+}-\left[\Lambda\left(H_{2}\right)\right]_{q} X_{1}^{+} X_{2}^{+}\right) X_{3}^{+} v_{0}+ \\
& +q^{-1} X_{3}^{+}\left(\left[\Lambda\left(H_{2}\right)-1\right]_{q} X_{1}^{+} X_{2}^{+}-\left[\Lambda\left(H_{2}\right)\right]_{q} X_{2}^{+} X_{1}^{+}\right) v_{0}= \\
= & q^{-1}\left(\left[\Lambda\left(H_{2}\right)+1\right]_{q} X_{2}^{+} X_{3}^{+}-\left[\Lambda\left(H_{2}\right)\right]_{q} X_{3}^{+} X_{2}^{+}\right) X_{1}^{+} v_{0}+ \\
& +q^{-1} X_{1}^{+}\left(-\left[\Lambda\left(H_{2}\right)-1\right]_{q} X_{1}^{+} X_{2}^{+}+\left[\Lambda\left(H_{2}\right)\right]_{q} X_{2}^{+} X_{1}^{+}\right) v_{0}
\end{aligned}
$$

where $[x]_{q} \equiv\left(q^{x}-q^{-x}\right) / \lambda, \lambda \equiv q-q^{-1}$.

Also for future reference we note several equivalent forms of the polynomial $\mathcal{P}$ valid for any weight:

$$
\begin{aligned}
\mathcal{P} & =X_{13}^{+} X_{2}^{+}-q^{-1} X_{12}^{+} X_{23}^{+}= \\
& =X_{13}^{+} X_{2}^{+}-q X_{23}^{+} X_{12}^{+}= \\
& =q^{-1}\left(X_{1}^{+} X_{2}^{+} X_{3}^{+} X_{2}^{+}+X_{2}^{+} X_{3}^{+} X_{2}^{+} X_{1}^{+}-[2]_{q} X_{2}^{+} X_{1}^{+} X_{3}^{+} X_{2}^{+}\right)= \\
& =q^{-1}\left(X_{3}^{+} X_{2}^{+} X_{1}^{+} X_{2}^{+}+X_{2}^{+} X_{1}^{+} X_{2}^{+} X_{3}^{+}-[2]_{q} X_{2}^{+} X_{1}^{+} X_{3}^{+} X_{2}^{+}\right)
\end{aligned}
$$

and two forms valid if $a \equiv \Lambda\left(H_{2}\right) \neq 1$ :

$$
\mathcal{P}=\frac{q^{-1}}{[a-1]_{q}}\left(X_{3}^{+} X_{2}^{+}-[2]_{q} X_{2}^{+} X_{3}^{+}\right)\left([a-1]_{q} X_{1}^{+} X_{2}^{+}-[a]_{q} X_{2}^{+} X_{1}^{+}\right)+
$$




$$
\begin{aligned}
& +\frac{1}{[a-1]_{q}} X_{2}^{+}\left([a-1]_{q} X_{1}^{+} X_{2}^{+}-[a]_{q} X_{2}^{+} X_{1}^{+}\right) X_{3}^{+}= \\
= & \frac{q^{-1}}{[a-1]_{q}}\left(X_{1}^{+} X_{2}^{+}-[2]_{q} X_{2}^{+} X_{1}^{+}\right)\left([a-1]_{q} X_{3}^{+} X_{2}^{+}-[a]_{q} X_{2}^{+} X_{3}^{+}\right)+ \\
& +\frac{1}{[a-1]_{q}} X_{2}^{+}\left([a-1]_{q} X_{3}^{+} X_{2}^{+}-[a]_{q} X_{2}^{+} X_{3}^{+}\right) X_{1}^{+}
\end{aligned}
$$

The need for the introduction of the parameter $a$ will become clear below.

2.5. Consider now a Verma module $V^{\Lambda}$ with lowest weight $\Lambda$ satisfying the conditions:

$$
\begin{aligned}
\Lambda\left(H_{3}\right) & =0 \Longleftrightarrow m_{3}=1 \\
\Lambda\left(H_{1}+H_{2}\right) & =1 \Longleftrightarrow m_{12}=1
\end{aligned}
$$

We shall denote its signature as:

$$
\chi_{1}(a)=\chi(\Lambda)=(a, 1-a, 1), \quad a=\Lambda\left(H_{2}\right) \in \mathbb{C}
$$

cf. $(2.9 c, d)$. We would like to study this family of representations (and a conjugate one) since for these the $(q-)$ d'Alembert operator will be a (conditionally) invariant operator. This will become clear in Sections 3 and 4 while here we find the necessary singular and subsingular vectors.

From the above two conditions follow that there are two singular vectors which are explicitly given by [6], [7]:

$$
\begin{aligned}
v_{3} & =X_{3}^{+} v_{0}, \quad m_{3}=1 \\
v_{12} & =\left([a-1]_{q} X_{1}^{+} X_{2}^{+}-[a]_{q} X_{2}^{+} X_{1}^{+}\right) v_{0}, \quad m_{12}=1
\end{aligned}
$$

(There is also a singular vector corresponding to $m_{13}=2[6],[7]$, which, however, is a descendant of $v_{3}$.)

In the above setting we shall show the special place of the the vector $v_{f}$ (which will give rise to the $(q-)$ d'Alembert operator as we shall see in the next sections). We have the following result:

If $a \neq 1$ the vector $v_{f}$ is a linear combination of descendants of the singular vectors $v_{3}$ and $v_{12}$, while if $a=1$ the vector $v_{f}$ is a subsingular vector.

It is straightforward to show this statement. Let first $a \neq 1$. Then using $(2.20 a)$ we have:

$$
\begin{aligned}
v_{f}=\mathcal{P} v_{0}= & \frac{q^{-1}}{[a-1]_{q}}\left(X_{3}^{+} X_{2}^{+}-[2]_{q} X_{2}^{+} X_{3}^{+}\right) v_{12}+ \\
& +\frac{1}{[a-1]_{q}} X_{2}^{+}\left([a-1]_{q} X_{1}^{+} X_{2}^{+}-[a]_{q} X_{2}^{+} X_{1}^{+}\right) v_{3}
\end{aligned}
$$

To show that for $a=1 v_{f}$ is a subsingular vector one may use a calculation valid for any $a$ (using also $(2.18 a))$ :

$$
\begin{aligned}
& X_{1}^{-} v_{f}=0 \\
& X_{2}^{-} v_{f}=q^{-1}\left([a+1]_{q} X_{2}^{+} X_{1}^{+}-[a]_{q} X_{1}^{+} X_{2}^{+}\right) v_{3}+q^{-1} X_{3}^{+} v_{12} \\
& X_{3}^{-} v_{f}=0
\end{aligned}
$$


though this calculations obscures the fact that for $a=1$ the singular vector $v_{12}$ is a descendant one, as we shall see below, where we also show that $v_{f}$ is not an element of $\tilde{I}^{\Lambda}$.

We now write down systematically all situations.

2.5.1. If $a \notin \mathbb{Z}$ there are no other nondescendant singular vectors, besides (2.22) and the maximal invariant submodule is: $I^{\Lambda}=I_{1}^{\prime}=I^{\alpha_{3}} \cup I^{\alpha_{12}}$. We denote by $L_{1}^{\prime}=V^{\Lambda} / I_{1}^{\prime}$ the corresponding irreducible factor-module, and by $\left|1^{\prime}\right\rangle$ the lowest weight vector of $L_{1}^{\prime}$. Then the expressions in (2.22) become null-conditions, namely we have:

$$
\begin{aligned}
X_{3}^{+}\left|1^{\prime}\right\rangle & =0 \\
\left([a-1]_{q} X_{1}^{+} X_{2}^{+}-[a]_{q} X_{2}^{+} X_{1}^{+}\right)\left|1^{\prime}\right\rangle & =0
\end{aligned}
$$

2.5.2. If $a \in-\mathbb{N}$ then in addition to (2.22) there is one more singular vector [6], [7] corresponding to $m_{2}=1-a \in \mathbb{N}+1$ :

$$
v_{2}=\left(X_{2}^{+}\right)^{1-a} v_{0}
$$

and two descendants corresponding to $m_{23}=2-a, m_{13}=2$. Thus the maximal invariant submodule is: $I^{\Lambda}=I_{1}^{\prime \prime}=I^{\alpha_{3}} \cup I^{\alpha_{12}} \cup I^{\alpha_{2}}, L_{1}^{\prime \prime}=V^{\Lambda} / I_{1}^{\prime \prime}$ is the irreducible factor-module, $\left|1^{\prime \prime}\right\rangle$ is the lowest weight vector of $L_{1}^{\prime \prime}$. Then the null-conditions are:

$$
\begin{aligned}
X_{3}^{+}\left|1^{\prime \prime}\right\rangle & =0 \\
\left([a-1]_{q} X_{1}^{+} X_{2}^{+}-[a]_{q} X_{2}^{+} X_{1}^{+}\right)\left|1^{\prime \prime}\right\rangle & =0 \\
\left(X_{2}^{+}\right)^{1-a}\left|1^{\prime \prime}\right\rangle & =0
\end{aligned}
$$

2.5.3. If $a=0$ then there is a singular vector corresponding to $m_{2}=1$ and given by (2.26) with $a=0$. Here also $(2.22 b)$ is descendant and the maximal invariant submodule is generated by the singular vectors $(2.22 a)$ and $(2.26), I^{\Lambda}=I_{1}^{\prime \prime \prime}=I^{\alpha_{3}} \cup I^{\alpha_{2}}$. We denote by $L_{1}^{\prime \prime \prime}=V^{\Lambda} / I_{1}^{\prime \prime \prime}$ the irreducible factor-module; $\left|1^{\prime \prime \prime}\right\rangle$ the lowest weight vector of $L_{1}^{\prime \prime \prime}$. Then the null-conditions are:

$$
\begin{aligned}
& X_{3}^{+}\left|1^{\prime \prime \prime}\right\rangle=0 \\
& X_{2}^{+}\left|1^{\prime \prime \prime}\right\rangle=0
\end{aligned}
$$

2.5.4. If $a \in \mathbb{N}+1$ then there exists another singular vector [6], [7]:

$$
v_{1}=\left(X_{1}^{+}\right)^{a} v_{0}
$$

Thus the maximal invariant submodule is: $I^{\Lambda}=I_{1}^{I V}=I^{\alpha_{3}} \cup I^{\alpha_{12}} \cup I^{\alpha_{1}}, L_{1}^{I V}=V^{\Lambda} / I_{1}^{I V}$ is the irreducible factor-module, $\left|1^{I V}\right\rangle$ is the lowest weight vector of $L_{1}^{I V}$. Then the null-conditions are:

$$
\begin{aligned}
X_{3}^{+}\left|1^{I V}\right\rangle & =0 \\
\left([a-1]_{q} X_{1}^{+} X_{2}^{+}-[a]_{q} X_{2}^{+} X_{1}^{+}\right)\left|1^{I V}\right\rangle & =0 \\
\left(X_{1}^{+}\right)^{a}\left|1^{I V}\right\rangle & =0
\end{aligned}
$$

2.5.5. Finally, if $a=1$ then the non-descendant singular vectors are $v_{3}=X_{3}^{+} v_{0}$, cf. $(2.22 a)$, and $v_{1}=X_{1}^{+} v_{0}$, cf. (2.29) with $a=1$, while $(2.22 b)$ is descendant of (2.29), and 
there appears also a singular vector $v_{23}^{\prime}$, cf. (2.11b), corresponding to $m_{23}=1$ which is descendant to $(2.22 a)$. Here we have also the subsingular vector $v_{f}$, cf. (2.16), (2.24), from the latter the essential one simplifying here to:

$$
X_{2}^{-} v_{f}=q^{-1}\left([2]_{q} X_{2}^{+} X_{1}^{+}-X_{1}^{+} X_{2}^{+}\right) v_{3}-q^{-1} X_{3}^{+} X_{2}^{+} v_{1}
$$

Now it remained from the proof above to show that $v_{f}$ can not be represented as a linear combination of descendants of $v_{1}$ and $v_{3}$, and thus does not belong to $\tilde{I}^{\Lambda}$, which is easy to see also by inspecting (2.19).

We denote by $\tilde{I}^{\Lambda}=I^{\alpha_{1}} \cup I^{\alpha_{3}}$ the submodule generated by these singular vectors, by $F_{1}=V^{\Lambda} / \tilde{I}^{\Lambda}$ the factor-module, by $\widetilde{|1\rangle}$ the lowest weight vector of $F_{1}$. We have the following null conditions in $F_{1}$ :

$$
\begin{aligned}
& X_{3}^{+} \widetilde{|1\rangle}=0 \\
& X_{1}^{+} \mid \widetilde{1\rangle}=0
\end{aligned}
$$

The vector $v_{f}$ becomes a singular vector in $F_{1}$ which we denote as:

$$
v_{f_{1}}=\mathcal{P} \mid \widetilde{1\rangle}
$$

Factoring out the submodule built on $v_{f_{1}}$ we obtain the irreducible factor-module $L_{1}=$ $V^{\Lambda} / I_{1}^{\Lambda}$. We denote by $|1\rangle$ the lowest weight vector of $L_{1}$. Then the null-conditions are:

$$
\begin{aligned}
X_{3}^{+}|1\rangle & =0 \\
X_{1}^{+}|1\rangle & =0 \\
\left(X_{3}^{+} X_{2}^{+}-[2]_{q} X_{2}^{+} X_{3}^{+}\right) X_{1}^{+} X_{2}^{+}|1\rangle & =0
\end{aligned}
$$

where for $(2.34 c)$ we have used $(2.34 a)$ and $(2.19 d)$. An equivalent condition to $(2.34 c)$ is:

$$
\left(X_{1}^{+} X_{2}^{+}-[2]_{q} X_{2}^{+} X_{1}^{+}\right) X_{3}^{+} X_{2}^{+}|1\rangle=0
$$

where we have used $(2.34 b)$ and $(2.19 c)$.

Conditions (2.30) and (2.34) $\left(\left(2.34 c, c^{\prime}\right)\right.$ in a different, but equivalent form) were given first in [14]. The corresponding irreps (for $a \in \mathbb{I}$ ) were shown [14] to be a construction of the irreducible massless representations of a $q$ - conformal algebra (with $|q|=1$ ) characterized by the helicity $h=(a-1) / 2 \in(1 / 2) \mathbb{Z}_{+}$.

2.6. Analogously consider a Verma module $V^{\Lambda}$ with lowest weight $\Lambda$ satisfying the conditions:

$$
\begin{aligned}
\Lambda\left(H_{1}\right) & =0 \Longleftrightarrow m_{1}=1 \\
\Lambda\left(H_{2}+H_{3}\right) & =1 \Longleftrightarrow m_{23}=1 \\
\chi_{3}(a) & =\chi(\Lambda)=(1,1-a, a), \quad a=\Lambda\left(H_{2}\right) \in \mathbb{C}
\end{aligned}
$$

cf. $(2.9 a, d)$. This case is conjugate to the one considered in the previous subsection and all statements and formulae may be obtained verbatim by exchanging indices $1 \longleftrightarrow 3$, $12 \longleftrightarrow 23$. Thus, we shall give for future reference only the final formulae analogous to (2.30). Namely, the conditions fulfilled in the irreducible lowest weight module $L_{3}$ (with 
$a \in \mathbb{N}+1)$ are:

$$
\begin{aligned}
X_{1}^{+}|3\rangle & =0 \\
\left([a-1]_{q} X_{3}^{+} X_{2}^{+}-[a]_{q} X_{2}^{+} X_{3}^{+}\right)|3\rangle & =0 \\
\left(X_{3}^{+}\right)^{a}|3\rangle & =0
\end{aligned}
$$

Conditions (2.36) were given first in [14].

It is interesting to note that a lowest weight can satisfy both (2.21) and (2.35) which happens only for the special case $a=1$, which was considered in the previous subsection.

\section{Conditionally invariant equations}

3.1. We now write down explicitly the conditionally invariant equations related to the subsingular vectors considered in the previous section. For simplicity we treat the case $q=1$ first and the $q$-deformed analogs in next Section.

We use the approach of [6] which we give in a condensed form here. We work with induced representations, called elementary representations (ERs). The functions of the ERs can be taken to be complex-valued $C^{\infty}$ functions on the group $G$. The representation action is given by the left regular action, which infinitesimally is:

$$
\left.\left(X_{L} \varphi\right)(g) \equiv \frac{d}{d t} \varphi(\exp (-t X) g)\right|_{t=0}
$$

where $X \in \mathcal{G}, g \in G, \mathcal{G}$ is the Lie algebra of $G$. These functions possess the properties of right covariance [6]. For our purposes it is enough to consider holomorphic elementary representations for which right covariance means:

$$
\begin{aligned}
& \hat{X} \varphi=\Lambda(X) \cdot \varphi, \quad X \in \mathcal{H} \\
& \hat{X} \varphi=0, \quad X \in \mathcal{G}^{-}
\end{aligned}
$$

where $\Lambda \in \mathcal{H}^{*}$, and $\hat{X}$ is the right action of the generators of the algebra $\mathcal{G}$

$$
\left.(\hat{X} \varphi)(g) \equiv \frac{d}{d t} \varphi(g \exp (t X))\right|_{t=0}
$$

Right covariance is used also to pass from functions on the group $G$ to the so-called reduced functions $\hat{\varphi}$ on the coset space $G / B$, where $B=\exp (\mathcal{H}) \exp \left(\mathcal{G}^{-}\right)$is a Borel subgroup of $G$. Note that $G / B$ is a completion of $G^{+}=\exp \left(\mathcal{G}^{+}\right)$and in practical calculations one is usually using the local coordinates of $G^{+}$.

The weight $\Lambda$ completely characterizes these representations, which we denote by $C^{\Lambda}$, each of which is then in correspondence with the lowest weight representations with the same lowest weight, in particular, with the Verma module $V^{\Lambda}$.

Now the main ingredient of the procedure of $[6]$ is that to every singular vector there corresponds an intertwining differential operator. Namely, to the singular vector $v_{s}=$ $v^{\alpha, m_{\alpha}}$ (cf. (2.5)) of the Verma module $V^{\Lambda}$ there corresponds an intertwining differential operator

$$
D^{\alpha, m_{\alpha}}: C^{\Lambda} \longrightarrow C^{\Lambda+m_{\alpha} \alpha}
$$

given explicitly by:

$$
D^{\alpha, m_{\alpha}}=\mathcal{P}^{\alpha, m}\left(\hat{X}_{1}^{+}, \ldots, \hat{X}_{r}^{+}\right)
$$


where $\mathcal{P}^{\alpha, m}$ is the same polynomial as in (2.5), and $\hat{X}_{i}^{+}$is the right action (3.3). This operator gives rise to the $\mathcal{G}$-invariant equation:

$$
D^{\alpha, m_{\alpha}} \hat{\varphi}=\hat{\varphi}^{\prime}, \quad \hat{\varphi} \in C^{\Lambda}, \hat{\varphi}^{\prime} \in C^{\Lambda+m_{\alpha} \alpha}
$$

In the same way a subsingular vector produces a differential operator and equation which are conditionally invariant. The latter means that this invariance hold only on the intersection of the kernels of all intertwining operators $D^{\alpha, m_{\alpha}}$ such that $\alpha$ and the singular vectors $v^{\alpha, m_{\alpha}}$ are associated with the singular vector in question, i.e., on the space:

$$
C_{\mathrm{su}}=\left\{\hat{\varphi} \in C^{\Lambda} \mid D^{\alpha, m_{\alpha}} \hat{\varphi}=0, \quad \forall \alpha \in \Delta_{\mathrm{su}}\right\}
$$

(cf. subsection 2.1.). A conditionally invariant equation has nontrivial RHS if we take the situation corresponding to the reducible factor-module $F^{\Lambda}=V^{\Lambda} / \tilde{I}^{\Lambda}$; the latter is realized when we do not impose in $F^{\Lambda}$ the null condition corresponding to the subsingular vector which in $F^{\Lambda}$ is a singular vector. A conditionally invariant equation has trivial RHS if we take the situation corresponding to the irreducible factor-module $L_{\Lambda}=V^{\Lambda} / I^{\Lambda}$, i.e., if we impose in $F^{\Lambda}$ the null condition corresponding to the subsingular vector. Below we consider both situations, for which we are prepared by the detailed analysis of Section 2 .

Remark 3. Note that one may exchange the left and right actions in the above considerations, i.e., consider the representations acting as right regular representations with properties of left covariance. Independently, if one uses highest weight representations (cf. Remark 1) one then uses the coset $G / B^{\prime}$, where $B^{\prime}=\exp (\mathcal{H}) \exp \left(\mathcal{G}^{+}\right)$is the Borel subgroup of $G$ conjugate to $B$. $\diamond$

Remark 4. As we noted if one wants to treat the case of a real noncompact algebra $\mathcal{G}_{0}$ one has to use also the results for its complexification $\mathcal{G}$. The application of these results to $\mathcal{G}_{0}$ has some subtleties [6]. However, in the case at hand when $\mathcal{G}_{0}=s u(2,2)$ and $\mathcal{G}=s l(4)$ the passage to $s u(2,2)$ is straightforward [5]. Also considering representations of the corresponding groups (which are used here only to provide the representation spaces) involves some subtleties [6], which, however, are not felt in the case under consideration [5]. $\diamond$

Referring further the general case to [6] we restrict here to $\mathcal{G}=\operatorname{sl}(4), G=S L(4)$. We pass to functions on the flag manifold $\mathcal{Y}=S L(4) / B$, where $B$ is the Borel subgroup of $S L(4)$ consisting of all upper diagonal matrices. (Equally well one may take the flag manifold $S L(4) / B^{\prime}$, where $B^{\prime}$ is the Borel subgroup of lower diagonal matrices.) We denote the six local coordinates on $\mathcal{Y}$ by $x_{ \pm}, v, \bar{v}, z, \bar{z}$. From the explicit form of the singular vectors it is clear that we need only the right action of the three simple root generators. Denoting this right action of $X_{k}^{+}$by $R_{k}$, we have from [6]:

$$
\begin{aligned}
& R_{1}=\partial_{z} \equiv \frac{\partial}{\partial z} \\
& R_{2}=\bar{z} z \partial_{+}+z \partial_{v}+\bar{z} \partial_{\bar{v}}+\partial_{-} \\
& R_{3}=\partial_{\bar{z}} \equiv \frac{\partial}{\partial \bar{z}}
\end{aligned}
$$


where

$$
\partial_{ \pm} \equiv \frac{\partial}{\partial x_{ \pm}}, \quad \partial_{v} \equiv \frac{\partial}{\partial v}, \quad \partial_{\bar{v}} \equiv \frac{\partial}{\partial \bar{v}},
$$

Things are arranged so that in the conformal setting we can use the same coordinates [5]. In this case the coordinates $x_{ \pm}, v, \bar{v}$ are related to the Minkowski space-time coordinates $x_{0}, x_{1}, x_{2}, x_{3}$ :

$$
x_{ \pm} \equiv x_{0} \pm x_{3}, \quad v \equiv x_{1}-i x_{2}, \quad \bar{v} \equiv x_{1}+i x_{2}
$$

while $z, \bar{z}$ encode the inducing Lorentz representation as explained below. In particular, one may use the following covariant representation for $R_{2}$ [5] employing the Pauli matrices $\sigma_{\mu}:$

$$
\begin{aligned}
& R_{2}=\left(\begin{array}{ll}
\bar{z} & 1
\end{array}\right) \sigma_{\mu} \partial^{\mu}\left(\begin{array}{l}
z \\
1
\end{array}\right) \\
& \sigma_{0}=\left(\begin{array}{ll}
1 & 0 \\
0 & 1
\end{array}\right), \quad \sigma_{1}=\left(\begin{array}{ll}
0 & 1 \\
1 & 0
\end{array}\right), \quad \sigma_{2}=\left(\begin{array}{cc}
0 & -i \\
i & 0
\end{array}\right), \quad \sigma_{3}=\left(\begin{array}{cc}
1 & 0 \\
0 & -1
\end{array}\right)
\end{aligned}
$$

Note also that under the natural conjugation:

$$
\omega\left(x_{ \pm}\right)=x_{ \pm}, \quad \omega(v)=\bar{v}, \quad \omega(z)=\bar{z}
$$

$\mathcal{Y}$ is also a flag manifold of the conformal group $S U(2,2)$.

The reduced function spaces of the ERs in which our equations are defined are complex-valued $C^{\infty}$ functions on the flag manifold. The holomorphic ERs of $s l(4)$ are labelled by the signature $\chi=\left(m_{1}, m_{2}, m_{3}\right)$.

In the $s u(2,2)$ case most applications in physics are in the case when $m_{1}, m_{3} \in \mathbb{N}$ and one uses reduced functions which are polynomials in the variables $z, \bar{z}$ of degrees $m_{1}-1, m_{3}-1$, resp. These then carry finite-dimensional irreducible representations of the Lorentz algebra of dimension $m_{1} m_{3}$. Let us stress that this is an indexless notation on which all Lorentz components of the fields are gathered together by the polynomial dependence in $z, \bar{z}$. To restore the components one has to take the entries of the independent terms in $z, \bar{z}$, cf. [5]. Note that in the physics literature often is used, instead of $\left(m_{1}, m_{2}, m_{3}\right)$, the labelling $\left[d, j_{1}, j_{2}\right]$, where $d=2-\left(m_{13}+m_{2}\right) / 2$ is the conformal weight, $j_{1}=\left(m_{1}-1\right) / 2, j_{2}=\left(m_{3}-1\right) / 2$, so that finite-dimensional Lorentz irreps one has: $j_{k} \in \mathbb{Z}_{+} / 2$.

3.2. We start with the equations arising from the $B G G$ example of subsingular vector. Substituting (3.8) in (2.14) we obtain the following $s l(4)$ and $s u(2,2)$ invariant equation:

$$
R_{2} \hat{\varphi}=\left(\bar{z} z \partial_{+}+z \partial_{v}+\bar{z} \partial_{\bar{v}}+\partial_{-}\right) \hat{\varphi}=0
$$

while the subsingular vector $v_{\mathrm{bgg}}$ gives rise to the following conditionally invariant equation:

$$
\left(R_{1} R_{2} R_{3}-R_{3} R_{2} R_{1}\right) \hat{\varphi}=\left(\partial_{v} \partial_{\bar{z}}-\partial_{\bar{v}} \partial_{z}+\left(\bar{z} \partial_{\bar{z}}-z \partial_{z}\right) \partial_{+}\right) \hat{\varphi}=\hat{\varphi}^{\prime}
$$

where $\hat{\varphi} \in C^{\Lambda}$ and satisfies (3.13a), $\hat{\varphi}^{\prime} \in C^{\Lambda^{\prime}}, \Lambda^{\prime}=\Lambda-\alpha_{13}$, the corresponding signatures being $\chi=(0,1,0), \chi^{\prime}=(-1,1,-1)$. (Note that the second Casimir operator has the same value in the two representations: $C_{2}(\chi)=C_{2}\left(\chi^{\prime}\right)=-4$, cf. (2.10).) If we consider the 
irreducible factor-module $L_{\Lambda}$, which means that we should use (2.15) instead of (2.14), we have instead of $(3.13 b)$ :

$$
\left(\partial_{v} \partial_{\bar{z}}-\partial_{\bar{v}} \partial_{z}+\left(\bar{z} \partial_{\bar{z}}-z \partial_{z}\right) \partial_{+}\right) \hat{\varphi}=0
$$

3.3. We pass now to equations arising from the other archetypal $s l(4)$ example. We consider the case when the lowest weight satisfies conditions (2.21).

We shall substitute the operators $R_{k}$ into the null conditions (2.25), (2.27), (2.28), (2.30), (2.32), (2.34). In all cases we have the equation arising from the singular vector $v_{3}=X_{3}^{+} v_{0}$, (null conditions $\left.(2.25 a),(2.27 a),(2.28 a),(2.30 a),(2.32 a),(2.34 a)\right)$ :

$$
R_{3} \hat{\varphi}=\partial_{\bar{z}} \hat{\varphi}=0
$$

which means that our functions do not depend on the variable $\bar{z}$ - this is valid for the signature $\chi_{1}(a)$ and arbitrary $a$. (In the conjugate situation with signature $\chi_{3}(a)$ our functions do not depend on the variable $z$.)

Further, we have the equations arising from the singular vector $v_{2}$, when $a \in \mathbb{Z}_{-}$, (null conditions $(2.27 c),(2.28 b))$ :

$$
\left(R_{2}\right)^{1-a} \hat{\varphi}=0, \quad a \in \mathbb{Z}_{-}
$$

Next, we have the equations arising from the singular vector $v_{1}$, when $a \in \mathbb{N}$, (null conditions $(2.30 c),(2.32 b),(2.34 b))$ :

$$
\left(\partial_{z}\right)^{a} \hat{\varphi}=0, \quad a \in \mathbb{N}
$$

which means that our functions are polynomials in the variable $z$ of degree $a-1$. Thus for $a=1$ our functions do not depend also on $z$.

Next we write down the equation arising from the singular vector $v_{12}$ (null conditions $(2.25 b),(2.27 b),(2.30 b))$ :

$$
\left((a-1) R_{1} R_{2}-a R_{2} R_{1}\right) \hat{\varphi}=\left((a-1)\left(\partial_{v}+\bar{z} \partial_{+}\right)-R_{2} \partial_{z}\right) \hat{\varphi}=0
$$

It is also valid in all cases, however, for $a=0$ it follows from (3.15) and for $a=1$ it follows from (3.16). Now, since (3.17) is a first degree polynomial in $\bar{z}$, on which our functions do not depend, it actually consists of two equations, though not invariant by themselves, i.e., we have:

$$
\begin{aligned}
& \left(\left(a-1-z \partial_{z}\right) \partial_{v}-\partial_{-} \partial_{z}\right) \hat{\varphi}=0 \\
& \left(\left(a-1-z \partial_{z}\right) \partial_{+}-\partial_{\bar{v}} \partial_{z}\right) \hat{\varphi}=0
\end{aligned}
$$

Finally, we obtain the conditionally invariant equations corresponding to the subsingular vector $v_{f}$. Let us denote by $\hat{\mathcal{P}}$ the polynomial $\mathcal{P}$ with $X_{k}^{+}$replaced by $R_{k}$. Now we shall obtain this operator in explicit form:

$$
\begin{aligned}
\hat{\mathcal{P}} \hat{\varphi} & =\left(R_{3} R_{2}-2 R_{2} R_{3}\right) R_{1} R_{2} \hat{\varphi}= \\
& =\left(z \partial_{+}+\partial_{\bar{v}}-R_{2} \partial_{\bar{z}}\right) \partial_{z} R_{2} \hat{\varphi}= \\
& =\left(\left(z \partial_{+}+\partial_{\bar{v}}\right) \partial_{z} R_{2}-R_{2} \partial_{z}\left(z \partial_{+}+\partial_{\bar{v}}\right)\right) \hat{\varphi}= \\
& =\left(\partial_{\bar{v}} \partial_{v}-\partial_{-} \partial_{+}\right) \hat{\varphi}=\square \hat{\varphi}
\end{aligned}
$$


where we used (3.14) in passing from $(3.19 b)$ to $(3.19 c)$. Thus, we have recovered the d'Alembert operator. Note that (3.19) is valid for arbitrary a since we have used only condition (3.14) which is valid for all of our representations.

Now for $a=1$ if we take only invariant equations arising from the conditions (2.32), (i.e., we work with the counterpart of the factor-module $F_{1}$ ), we have the following system of differential equations:

$$
\begin{aligned}
\partial_{\bar{z}} \hat{\varphi} & =0 \\
\partial_{z} \hat{\varphi} & =0 \\
\square \hat{\varphi} & =\hat{\varphi}^{\prime}
\end{aligned}
$$

where $\hat{\varphi} \in C^{\Lambda}$ and satisfies $(3.20 a, b), \hat{\varphi}^{\prime} \in C^{\Lambda^{\prime}}, \Lambda^{\prime}=\Lambda-\alpha_{13}-\alpha_{2}$, the corresponding signatures being $\chi=(1,0,1), \chi^{\prime}=(1,-2,1)$. (Note that the second Casimir operator has the same value in the two representations: $C_{2}(\chi)=C_{2}\left(\chi^{\prime}\right)=-3$, cf. (2.10).) If we consider the irreducible factor-module $L_{1}$, which means that we should use (2.34) instead of $(2.32)$, we have instead of $(3.20 c)$ :

$$
\square \hat{\varphi}=0
$$

where $\hat{\varphi}$ is as in $(3.20 c)$ and again satisfies $(3.20 a, b)$.

Thus, from the subsingular vector $v_{f}$ we have obtained the d'Alembert equations $(3.20 c, d)$ as conditionally $\operatorname{sl}(4)$ and $s u(2,2)$ invariant equations.

Now we pass to the cases when $a \neq 1$. In these cases the vector $v_{f}$ is a linear combination of the singular vectors $v_{1}$ and $v_{12}$ and it becomes zero when these singular vectors are factorized. Since $v_{f}$ gives rise to the d'Alembert operators for all $a$ we expect that the d'Alembert equation $(3.20 d)$ will hold automatically if the invariant equations (3.14), (3.17) (arising from $v_{1}, v_{12}$ ) hold. This is indeed so. We use the two equations (3.18) which are the two components of (3.17). First we take $\partial_{\bar{v}}$ derivative from $(3.18 a)$ and $\partial_{-}$ derivative from $(3.18 b)$ and subtracting the two we get:

$$
\left(a-1-z \partial_{z}\right)\left(\partial_{-} \partial_{+}-\partial_{\bar{v}} \partial_{v}\right) \hat{\varphi}=\left(a-1-z \partial_{z}\right) \square \hat{\varphi}=0
$$

This still follows from $(3.20 d)$. Analogously, taking $\partial_{+}$derivative from $(3.18 a)$ and $\partial_{v}$ derivative from $(3.18 b)$ and subtracting the two we get:

$$
\partial_{z}\left(\partial_{-} \partial_{+}-\partial_{\bar{v}} \partial_{v}\right) \hat{\varphi}=\partial_{z} \square \hat{\varphi}=0
$$

This also follows from $(3.20 d)$. Now, clearly from $(3.21 a, b)$ follows:

$$
(a-1) \square \hat{\varphi}=0
$$

which implies the d'Alembert equation if $a \neq 1$.

Using the conjugate situation with signature $\chi_{3}(a)$ we recover the d'Alembert equation on functions which do not depend on $z$ and satisfy

$$
\begin{aligned}
& \left((a-1) R_{3} R_{2}-a R_{3} R_{1}\right) \hat{\varphi}= \\
& =\left((a-1)\left(\partial_{\bar{v}}+z \partial_{+}\right)-R_{2} \partial_{\bar{z}}\right) \hat{\varphi}=0
\end{aligned}
$$


instead of (3.17). Furthermore the analogs of $(3.18 a, b),(3.14),(3.16)$, resp., are:

$$
\begin{aligned}
\left(\left(a-1-\bar{z} \partial_{\bar{z}}\right) \partial_{\bar{v}}-\partial_{-} \partial_{\bar{z}}\right) \hat{\varphi} & =0 \\
\left(\left(a-1-\bar{z} \partial_{\bar{z}}\right) \partial_{+}-\partial_{v} \partial_{\bar{z}}\right) \hat{\varphi} & =0 \\
\partial_{z} \hat{\varphi} & =0 \\
\left(\partial_{\bar{z}}\right)^{a} \hat{\varphi} & =0, \quad a \in \mathbb{N}
\end{aligned}
$$

Thus if $a \in \mathbb{N}$, then the functions of the irreducible representations are polynomials in $\bar{z}$ of degree $a-1$.

If $a \in \mathbb{Z}_{-}$our functions satisfy (3.15) as those with signature $\chi_{1}(a)$.

Finally the d'Alembert equation $(3.20 d)$ follows from equations $(3.23 a, b)(a \neq 1)$. We do not need to consider $a=1$ since the two signatures coincide.

We summarize now the results of this subsection. The first result is that the d'Alembert equation $(1.1),(3.20 d)$ holds in the representation spaces with signatures $\chi_{1}(a)=$ $(a, 1-a, 1)$, resp., $\chi_{3}(a)=(1,1-a, a)$, if our functions do not depend on the variable $\bar{z}$, resp., $z$ and in addition satisfy $(3.20 a, b)$, resp., $(3.23 a, b)$. For $a=1$ the d'Alembert equations $(3.20 c, d)$ are conditionally $s l(4)$ and $s u(2,2)$ invariant, while for $a \neq 1$ the d'Alembert equation (3.20d) just follows from $(3.20 a, b)$, resp., $(3.23 a, b)$. If $a \in \mathbb{I}$ then our functions are polynomials in $z$, resp., $\bar{z}$, of degree $a-1$.

In the $s u(2,2)$ setting we again recall that the variables $z, \bar{z}$ are representing the spin dependence coming from the Lorentz representation [5], [11], [12]. The above result then is restated so in the case $a \notin \mathbb{N}$ : the d'Alembert equation holds if the fields carry holomorphic (depending only on $z$ ) or antiholomorphic (depending only on $\bar{z}$ ) infinitedimensional representations of the Lorentz algebra; in addition they satisfy $(3.20 a, b)$, resp., $(3.23 a, b)$. In the case $a \in \mathbb{N}$ we restrict to Lorentz representations which are finite-dimensional; in fact, of dimension $a$.

The case $a=1$ is remarkable in one more respect, namely, in this case one may have a nontrivial RHS, cf. (3.20c). It is easy to check that there are no other cases with nontrivial RHS. In fact, for $a \neq 1(3.20 d)$ follows from $(3.20 a, b)$, or $(3.23 a, b)$. This can be shown also independently. Indeed, in the first case the candidate signatures would be: $\chi_{1}(a)=(a, 1-a, 1), \chi_{1}^{\prime}(a)=(a,-1-a, 1)$. We know that a necessary condition to have an invariant equation is that the two representations would have the same Casimir operators, in particular, one should have $C_{2}\left(\chi_{1}(a)\right)-C_{2}\left(\chi_{1}^{\prime}(a)\right)=0$, where $C_{2}$ is given in (2.10). Calculating this difference we obtain:

$$
C_{2}\left(\chi_{1}(a)\right)-C_{2}\left(\chi_{1}^{\prime}(a)\right)=2(a-1)
$$

which is not zero unless $a=1$.

The cases $a>1$ are interesting in other contexts, especially, if we consider together the representations with the conjugated signatures $\chi_{1}(a)$ and $\chi_{3}(a)$ with the same $a \in \mathbb{I}+1$. In particular, in the case $a=2$ the two conjugated fields are two-component spinors and (3.17), (3.22) are the two conjugated Weyl equations.

The cases $a=3$ are maybe most interesting. The Lorentz dimension is $6(=2 a)$ and the resulting field is the Maxwell field. As was shown in detail in [11] equations (3.17), 
(3.22) are just a rewriting of the free Maxwell equations:

$$
\partial^{\mu} F_{\mu \nu}=0, \quad \partial^{\mu *} F_{\mu \nu}=0
$$

Remark 5. Actually, in [11] were considered the general Maxwell equations with nonzero current, i.e.,

$$
\partial^{\mu} F_{\mu \nu}=J_{\nu}, \quad \partial^{\mu *} F_{\mu \nu}=0
$$

which are then equivalent to a modification of (3.17), (3.22) with nonzero right-hand-sides which are given explicitly in formulae $(5 \mathrm{a}, \mathrm{b})$ of [11]. More than that, in [11] is discussed an hierarchy of Maxwell equations involving two conjugated families of representations: $\chi_{n}^{+}=(n+3,-n-2, n+1), \chi_{n}^{-}=(n+1,-n-2, n+3), n \in \mathbb{Z}_{+}$, from which the Maxwell case is obtained for $n=0$. Note that there is no other intersection of this Maxwell hierarchy with the two families $\chi_{1}(a)$ and $\chi_{3}(a)$ (cf. $(2.21 c),(2.35 c)$ ) which we consider in the present paper. $\diamond$

We may write out many other equations with indices, however, one of the main points here is that in this form equations (3.20) and (3.23) are valid different representation spaces, the different representations manifesting themselves only through the parameter $a$.

Remark 6.1. It is interesting to note that there are other conditionally invariant equations involving the d'Alembert operator, from which $(3.20 c)$ is a partial case $(m=1)$, namely:

$$
\square^{m} \hat{\varphi}=\hat{\varphi}^{\prime}, \quad m \in \mathbb{N}
$$

where $\hat{\varphi} \in C^{\Lambda}, \hat{\varphi}^{\prime} \in C^{\Lambda^{\prime}}, \Lambda^{\prime}=\Lambda-m\left(\alpha_{13}+\alpha_{2}\right)$, the corresponding signatures being $\chi=(m, 0, m), \chi^{\prime}=(m,-2 m, m)$. These are produced by subsingular vectors of weights $m\left(\alpha_{13}+\alpha_{2}\right)$, [13]. The functions $\hat{\varphi}, \hat{\varphi}^{\prime}$ carry irreducible Lorentz representations which are symmetric traceless tensors of rank $m-1$. (For early examples, namely, (3.27) with $m=2$, obtained from other considerations, cf. [21], [3], [17].)

Rem ark 6.2. We should note that there are conditionally invariant equations involving the d'Alembert operator, which do not arise from subsingular vectors but from reduction of integral intertwining operators. These equations are also given by (3.27), however, the corresponding signatures are: $\chi=(m, n, m), \chi^{\prime}=(m, n-2 m, m), m, n \in \mathbb{N}$, cf., e.g., [16].

Remark 6.3. We should note that in (most of) the physical applications (3.27) is not considered conditionally invariant. The reason is that there only representations induced from finite-dimensional Lorentz representations are considered. The fact that these representations are also subspaces of reducible representations is ignored and thus the restriction to these subspaces is not considered to be a condition (cf. [20], [21], [3], [17], [16]).

\section{Conditionally invariant $q$-difference equations}

4.1. We give now the treatment of the conditionally invariant equations in the $q$ deformed case. We need first to introduce our reduced representation spaces $C^{\Lambda}$ with signatures $\chi=\chi(\Lambda)=\left(m_{1}, m_{2}, m_{3}\right)$, cf. [10], [11]. The elements of $C^{\Lambda}$, which we 
shall call (abusing the notion) functions, are formal power series in the noncommuting variables $z, v, x_{-}, x_{+}, \bar{v}, \bar{z}$, which generate the $q$-deformation $\mathcal{Y}_{q}$ of the flag manifold $\mathcal{Y}$ (the commutation relations of these variables using the same notation are given in [11]). More explicitly, these reduced functions are given by:

$$
\begin{aligned}
\hat{\varphi}(\bar{Y}) & =\sum_{i, j, k, \ell, m, n \in \mathbb{Z}_{+}} \mu_{i j k \ell m n} \hat{\varphi}_{i j k \ell m n} \\
\hat{\varphi}_{i j k \ell m n} & =z^{i} v^{j} x_{-}^{k} x_{+}^{\ell} \bar{v}^{m} \bar{z}^{n}
\end{aligned}
$$

where $\bar{Y}$ denotes the set of the six variables.

Next we introduce the following operators acting on our functions:

$$
\begin{aligned}
\hat{M}_{\kappa} \hat{\varphi}(\bar{Y}) & =\sum_{i, j, k, \ell, m, n \in \mathbb{Z}_{+}} \mu_{i j k \ell m n} \hat{M}_{\kappa} \hat{\varphi}_{i j k \ell m n}, \\
T_{\kappa} \hat{\varphi}(\bar{Y}) & =\sum_{i, j, k, \ell, m, n \in \mathbb{Z}_{+}} \mu_{i j k \ell m n} T_{\kappa} \hat{\varphi}_{i j k \ell m n},
\end{aligned}
$$

where $\kappa=z, \pm, v, \bar{v}, \bar{z}$, and the explicit action on $\hat{\varphi}_{i j k \ell m n}$ is defined by:

$$
\begin{aligned}
\hat{M}_{z} \hat{\varphi}_{i j k \ell m n} & =\hat{\varphi}_{i+1, j k \ell m n}, \\
\hat{M}_{v} \hat{\varphi}_{i j k \ell m n} & =\hat{\varphi}_{i, j+1, k \ell m n}, \\
\hat{M}_{-} \hat{\varphi}_{i j k \ell m n} & =\hat{\varphi}_{i j, k+1, \ell m n}, \\
\hat{M}_{+} \hat{\varphi}_{i j k \ell m n} & =\hat{\varphi}_{i j k \ell \ell+1, m n}, \\
\hat{M}_{\bar{v}} \hat{\varphi}_{i j k \ell m n} & =\hat{\varphi}_{i j k \ell, m+1, n}, \\
\hat{M}_{\bar{z}} \hat{\varphi}_{i j k \ell m n} & =\hat{\varphi}_{i j k \ell m, n+1}, \\
T_{z} \hat{\varphi}_{i j k \ell m n} & =q^{i} \hat{\varphi}_{i j k \ell m n}, \\
T_{v} \hat{\varphi}_{i j k \ell m n} & =q^{j} \hat{\varphi}_{i j k \ell m n}, \\
T_{-} \hat{\varphi}_{i j k \ell m n} & =q^{k} \hat{\varphi}_{i j k \ell m n}, \\
T_{+} \hat{\varphi}_{i j k \ell m n} & =q^{\ell} \hat{\varphi}_{i j k \ell m n}, \\
T_{\bar{v}} \hat{\varphi}_{i j k \ell m n} & =q^{m} \hat{\varphi}_{i j k \ell m n}, \\
T_{\bar{z}} \hat{\varphi}_{i j k \ell m n} & =q^{n} \hat{\varphi}_{i j k \ell m n} .
\end{aligned}
$$

Now we define the $q$-difference operators by:

$$
\hat{\mathcal{D}}_{\kappa} \hat{\varphi}(\bar{Y})=\frac{1}{\lambda} \hat{M}_{\kappa}^{-1}\left(T_{\kappa}-T_{\kappa}^{-1}\right) \hat{\varphi}(\bar{Y}), \quad \kappa=z, \pm, v, \bar{v}, \bar{z} .
$$

Note that although $\hat{M}_{\kappa}^{-1}$ is not defined if the corresponding variable is of zero degree, the operator $\hat{\mathcal{D}}_{\kappa}$ is well defined on such terms, and the result is zero (given by the action of $\left.\left(T_{\kappa}-T_{\kappa}^{-1}\right)\right)$. Of course, for $q \rightarrow 1$ we have $\hat{\mathcal{D}}_{\kappa} \rightarrow \partial_{\kappa}$.

Using the above operators the representation (left) action was given in [10] for general $n$ and for $n=4$ in [12].

The $q$-difference analogues of the operators $R_{k}$, i.e., the right action of $U_{q}(s l(4))$ on our functions is also known from [10]. Adapting this to our notation we have :

$$
R_{1}^{q}=\hat{\mathcal{D}}_{z} T_{z}\left(T_{v} T_{-} T_{+} T_{\bar{v}}\right)^{-1}
$$




$$
\begin{aligned}
R_{2}^{q}= & \left(q \hat{M}_{z} \hat{\mathcal{D}}_{v} T_{v}^{2} T_{-}^{2}+\hat{\mathcal{D}}_{-} T_{-}+\right. \\
& +\hat{M}_{z} \hat{M}_{\bar{z}} \hat{\mathcal{D}}_{+}\left(T_{v} T_{-}\right)^{-1} T_{\bar{v}}+q^{-1} \hat{M}_{\bar{z}} \hat{\mathcal{D}}_{\bar{v}}- \\
& \left.-\lambda \hat{M}_{v} \hat{M}_{\bar{z}} \hat{\mathcal{D}}_{-} \hat{\mathcal{D}}_{+} T_{\bar{v}}\right) T_{\bar{v}} T_{\bar{z}}^{-1} \\
R_{3}^{q}= & \hat{\mathcal{D}}_{\bar{z}} T_{\bar{z}}
\end{aligned}
$$

To obtain the (conditionally) invariant $q$-difference equations amounts now simply to substitute $X_{k}^{+}$with $R_{k}^{q}$ in the expressions of the (sub)singular vectors for general $q$.

4.2. Substituting (4.6) in (2.14) we obtain the following $U_{q}(s l(4))$ and $U_{q}(s u(2,2))$ invariant equation:

$$
\begin{aligned}
R_{2}^{q} \hat{\varphi}= & \left(q \hat{M}_{z} \hat{\mathcal{D}}_{v} T_{v}^{2} T_{-}^{2}+\hat{\mathcal{D}}_{-} T_{-}+\right. \\
& +\hat{M}_{z} \hat{M}_{\bar{z}} \hat{\mathcal{D}}_{+}\left(T_{v} T_{-}\right)^{-1} T_{\bar{v}}+q^{-1} \hat{M}_{\bar{z}} \hat{\mathcal{D}}_{\bar{v}}- \\
& \left.-\lambda \hat{M}_{v} \hat{M}_{\bar{z}} \hat{\mathcal{D}}_{-} \hat{\mathcal{D}}_{+} T_{\bar{v}}\right) T_{\bar{v}} T_{\bar{z}}^{-1} \hat{\varphi}=0
\end{aligned}
$$

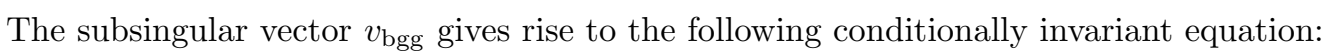

$$
\begin{aligned}
& \left(R_{1}^{q} R_{2}^{q} R_{3}^{q}-R_{3}^{q} R_{2}^{q} R_{1}^{q}\right) \hat{\varphi}= \\
& =\left\{q^{4} \hat{\mathcal{D}}_{v} \hat{\mathcal{D}}_{\bar{z}} T_{z}^{2} T_{v}^{2} T_{-}^{2}-\hat{\mathcal{D}}_{\bar{v}} \hat{\mathcal{D}}_{z} T_{z} T_{\bar{z}}+\right. \\
& +q^{2} \hat{\mathcal{D}}_{+} T_{+} T_{\bar{v}}^{2}\left(T_{z} T_{\bar{z}}+\left(q^{-1} T_{\bar{z}} T_{z}^{-1}-q T_{z} T_{\bar{z}}^{-1}\right) / \lambda\right)+ \\
& +q \lambda \hat{M}_{v} \hat{\mathcal{D}}_{-} \hat{\mathcal{D}}_{+} \hat{\mathcal{D}}_{z} T_{z} T_{\bar{z}} T_{\bar{v}}+ \\
& +q \lambda\left(q \hat{M}_{z} \hat{\mathcal{D}}_{v} T_{v}^{2} T_{-}^{2}+\hat{\mathcal{D}}_{-} T_{-}+\right. \\
& +\hat{M}_{z} \hat{M}_{\bar{z}} \hat{\mathcal{D}}_{+}\left(T_{v} T_{-}\right)^{-1} T_{\bar{v}}+q^{-1} \hat{M}_{\bar{z}} \hat{\mathcal{D}}_{\bar{v}-} \\
& \left.\left.-\lambda \hat{M}_{v} \hat{M}_{\bar{z}} \hat{\mathcal{D}}_{-} \hat{\mathcal{D}}_{+} T_{\bar{v}}\right) \hat{\mathcal{D}}_{\bar{z}} \hat{\mathcal{D}}_{z} T_{z}\right\}\left(T_{v} T_{-} T_{+}\right)^{-1} \hat{\varphi}=\hat{\varphi}^{\prime}
\end{aligned}
$$

where $\hat{\varphi}, \in C^{\Lambda}$ and satisfies $(4.7 a), \hat{\varphi}^{\prime} \in C^{\Lambda^{\prime}}, \Lambda^{\prime}=\Lambda-\alpha_{13}$, the corresponding signatures being as in (3.13). Clearly $(4.7 a, b)$ go into $(3.13 a, b)$ for $q=1$. If we consider the irreducible factor-module $L_{\Lambda}$, which means that we should use (2.15) instead of (2.14), then we have a zero RHS in (4.7b) as in (3.13c).

4.3. Finally, we write down the $q$-difference analogues of the d'Alembert equation and of the equations ensuring its $U_{q}(s l(4))$ and $U_{q}(s u(2,2))$ invariance and from which it follows (except for $a=1$ ). Substituting (4.6) in (2.34) we obtain:

$$
\begin{aligned}
& R_{3} \hat{\varphi}=\hat{\mathcal{D}}_{\bar{z}} T_{\bar{z}} \hat{\varphi}=0 \\
& \left([a-1]_{q} R_{1}^{q} R_{2}^{q}-[a]_{q} R_{2}^{q} R_{1}^{q}\right) \hat{\varphi}= \\
& =\left(q^{2}[a-1]_{q}\left(q \hat{\mathcal{D}}_{v} T_{v}^{2} T_{-}^{2}+\hat{M}_{\bar{z}} \hat{\mathcal{D}}_{+}\left(T_{v} T_{-}\right)^{-1} T_{\bar{v}}\right) T_{z}-\right.
\end{aligned}
$$




$$
\begin{aligned}
& -q^{1-a}\left(q \hat{M}_{z} \hat{\mathcal{D}}_{v} T_{v}^{2} T_{-}^{2}+\hat{\mathcal{D}}_{-} T_{-}+\right. \\
& +\hat{M}_{z} \hat{M}_{\bar{z}} \hat{\mathcal{D}}_{+}\left(T_{v} T_{-}\right)^{-1} T_{\bar{v}}+q^{-1} \hat{M}_{\bar{z}} \hat{\mathcal{D}}_{\bar{v}}- \\
& \left.\left.-\lambda \hat{M}_{v} \hat{M}_{\bar{z}} \hat{\mathcal{D}}_{-} \hat{\mathcal{D}}_{+} T_{\bar{v}}\right) \hat{\mathcal{D}}_{z}\right) T_{z}\left(T_{v} T_{-} T_{+}\right)^{-1} \hat{\varphi}=0 \\
& \left(R_{3}^{q} R_{2}^{q}-[2]_{q} R_{2}^{q} R_{3}^{q}\right) R_{1}^{q} R_{2}^{q} \hat{\varphi}=0
\end{aligned}
$$

As in the $q=1$ case we use (4.8a) to split (4.8b) in two equations and to simplify (4.8c). Finally, we have:

$$
\begin{aligned}
& \hat{\mathcal{D}}_{\bar{z}} T_{\bar{z}} \hat{\varphi}=0 \\
& \left(q^{a+2}[a-1]_{q} \hat{\mathcal{D}}_{v} T_{z} T_{v}^{2} T_{-}\right. \\
& \left.-\left(q \hat{M}_{z} \hat{\mathcal{D}}_{v} T_{v}^{2} T_{-}+\hat{\mathcal{D}}_{-}\right) \hat{\mathcal{D}}_{z}\right) T_{z}\left(T_{v} T_{+}\right)^{-1} \hat{\varphi}=0 \\
& \left(q^{a+1}[a-1]_{q} \hat{\mathcal{D}}_{+} T_{z}\left(T_{v} T_{-}\right)^{-1} T_{\bar{v}-}\right. \\
& -\left(\hat{M}_{z} \hat{\mathcal{D}}_{+}\left(T_{v} T_{-}\right)^{-1} T_{\bar{v}}+q^{-1} \hat{\mathcal{D}}_{\bar{v}-}\right. \\
& \left.\left.-\lambda \hat{M}_{v} \hat{\mathcal{D}}_{-} \hat{\mathcal{D}}_{+} T_{\bar{v}}\right) \hat{\mathcal{D}}_{z}\right) T_{z}\left(T_{v} T_{-} T_{+}\right)^{-1} \hat{\varphi}=0 \\
& \left\{\left(\hat{\mathcal{D}}_{v} \hat{\mathcal{D}}_{\bar{v}} T_{v}^{3} T_{\bar{v}}^{-1}-q \hat{\mathcal{D}}_{-} \hat{\mathcal{D}}_{+} T_{-}^{-2}\right) T_{z} T_{v}^{-1}-\right. \\
& \left.-q \hat{\mathcal{D}}_{-} \hat{\mathcal{D}}_{+}\left(T_{v} T_{z}^{-1}-T_{v}^{-1} T_{z}\right)\right\}\left(T_{v} T_{+}\right)^{-1} T_{z} T_{-} T_{\bar{v}}^{2} \hat{\varphi}=0
\end{aligned}
$$

In addition, if $a \in \mathbb{N}$ we have also:

$$
\left(\hat{\mathcal{D}}_{z} T_{z}\left(T_{v} T_{-} T_{+} T_{\bar{v}}\right)^{-1}\right)^{a} \hat{\varphi}=0
$$

In the scalar case $a=1$ the relevant equations are $(4.9 a, c, d)$, in particular, using $(4.9 c)$ and adding a nontrivial RHS we obtain the conditionally $U_{q}(s l(4))$ and $U_{q}(s u(2,2))$ invariant $q$-d'Alembert equation:

$$
\begin{aligned}
& \left\{\left(\hat{\mathcal{D}}_{v} \hat{\mathcal{D}}_{\bar{v}} T_{v}^{3} T_{\bar{v}}^{-1}-q \hat{\mathcal{D}}_{-} \hat{\mathcal{D}}_{+} T_{-}^{-2}\right) T_{v}^{-1}-\right. \\
& \left.-q \lambda \hat{\mathcal{D}}_{-} \hat{\mathcal{D}}_{+} \hat{M}_{v} \hat{\mathcal{D}}_{v}\right\}\left(T_{v} T_{+}\right)^{-1} T_{-} T_{\bar{v}}^{2} \hat{\varphi}=\hat{\varphi}^{\prime}, \quad a=1
\end{aligned}
$$

Analogously one may write down explicitly the conjugated invariant equations.

Clearly, for $q=1(4.9 c, e)$ go into the d'Alembert equations $(3.20 d, c)$, resp.

Acknowledgments. The author was supported in part by BNFR under contract $\mathrm{Ph}-401$. 


\section{References}

[1] A.O. Barut and R. Rączka, Theory of Group Representations and Applications, II edition, (Polish Sci. Publ., Warsaw, 1980).

[2] I.N. Bernstein, I.M. Gel'fand and S.I. Gel'fand, Funkts. Anal. Prilozh. 5 (1) (1971) 1; English translation: Funct. Anal. Appl. 5 (1971) 1.

[3] B. Binegar, C. Fronsdal and W. Heidenreich, J. Math. Phys. 24 (1983) 2828.

[4] C. De Concini and V.G. Kac, Progress in Math. 92 (Birkhäuser, Boston, 1990) p. 471.

[5] V.K. Dobrev, J. Math. Phys. 26 (1985) 235.

[6] V.K. Dobrev, Rep. Math. Phys. 25 (1988) 159.

[7] V.K. Dobrev, in: Proc. of the International Group Theory Conference (St. Andrews, 1989), eds. C.M. Campbell and E.F. Robertson, Vol. 1, London Math. Soc. Lect. Note Ser. 159 (1991) p. 87.

[8] V.K. Dobrev, Lett. Math. Phys. 22 (1991) 251.

[9] V.K. Dobrev, J. Phys. A26 (1993) 1317.

[10] V.K. Dobrev, J. Phys. A27 (1994) 4841 \& 6633, (first as preprint ASI-TPA/10/93 (1993)).

[11] V.K. Dobrev, Phys. Lett. 341B (1994) 133 \& 346B (1995) 427 (first as preprint ASITPA/15/94 (June 1994)).

[12] V.K. Dobrev, preprint ASI-TPA/22/94, (September 1994); invited talk at the Symposium "Symmetries in Science VIII", (August 1994, Bregenz, Austria), Proceedings, ed. B. Gruber, (Plenum Press, NY, 1995) p. 55.

[13] V.K. Dobrev, Kazhdan-Lusztig polynomials and subsingular vectors, ICTP preprint IC/96/15 (January 1996).

[14] V.K. Dobrev and R. Floreanini, J. Phys. A27 (1994) 4831.

[15] V.G. Drinfeld, Soviet. Math. Dokl. 32 (1985) 2548; in: Proceedings of the International Congress of Mathematicians, Berkeley (1986), Vol. 1 (The American Mathematical Society, Providence, 1987) p. 798.

[16] P. Furlan, V.B. Petkova and G.M. Sotkov, J. Math. Phys. 28 (1987) 251.

[17] P. Furlan, V.B. Petkova, G.M. Sotkov and I.T. Todorov, Riv. del Nuovo Cim. 8 No 3 (1985) 1 .

[18] M. Jimbo, Lett. Math. Phys. 10 (1985) 63; Lett. Math. Phys. 11 (1986) 247.

[19] D. Kazhdan and G. Lusztig, Inv. Math. 53 (1979) 165.

[20] Abdus Salam and J. Strathdee, Phys. Rev. 184 (1969) 1760.

[21] G.M. Sotkov and D.Ts. Stoyanov, J. Phys. A13 (1980) 2807; J. Phys. A16 (1983) 2817.

[22] T. Yao, J. Math. Phys. 8 (1967) 1931; 9 (1968) 1615; 12 (1971) 315. 